\title{
AGRESSIONS SEXUELLES \\ ET DÉPENDANCE À L'ADOLESCENCE
}

Jean-Yves Chagnon

Jean-Yves Chagnon

Professeur de

psychologie

clinique et de

psychopathologie,

UTRPP, Université

Paris 13, Sorbonne

Paris Cité ;

Psychologue

clinicien,

psychanalyste, expert judiciaire, membre du CILA.
RESUME : L’article, après quelques rappels épidémiologiques et juridiques permettant de cerner la problématique en cause, présente les grandes caractéristiques cliniques et psychopathologiques des adolescents agresseurs sexuels français. Puis l'exposé d'un cas d'adolescent violeur rencontré dans un cadre expertal soutient la proposition de quelques jalons théoriques relatifs à la place de l'agir dit «sexuel » dans le temps de l'adolescence et ses rapports à la problématique de dépendance. L’article se termine par quelques propos cursifs sur les incidences thérapeutiques posées par la compréhension de ce type d'agirs, inscrits dans ce que nous appelons aujourd'hui « les cliniques de l'extrême ».

Mots clés : Adolescence, agressions sexuelles, dépendance, identification projective, réalité externe.

ABSTRACT: Sexual aggressions and dependence during adolescence. After a brief reminder of the underlying epidemiological and legal context, this article presents an overview of the clinical and psychopathological characteristics of French adolescents who have committed sexual aggressions. The author presents the case of a young sex offender met in the framework of a psychological assessment report. The results provide support for the introduction of several theoretical contributions concerning the role of "sexual" acting-out during adolescence and its connection with dependence. The article concludes with a few remarks concerning the therapeutic implications of this analysis within the field of what is today called the "clinical study of extreme situations".

Keywords: Adolescence, sexual aggressions, dependence, projective identification, external reality. 


\section{INTRODUCTION}

En France les violences à l'adolescence sont depuis une vingtaine d'années (le tournant des années 90) l'objet d'une focalisation et d'une dramatisation sociale, politique et médiatique ayant justifié une progressive mutation des orientations de la Justice des Mineurs, les préoccupations sécuritaires des différents gouvernements ayant entrainé de multiples remaniements de l'Ordonnance dite de 1945. Parmi ces violences les violences sexuelles à l'adolescence occupent une situation particulière, de fascination ambigüe aux plans médiatique et sociétal, de réelle préoccupation aux plans épidémiologique, clinique, psychopathologique, judiciaire et thérapeutique ; elles interrogent enfin les rapports entre les individus, les sexes et les générations dans un temps — celui de l'hypermodernité - caractérisé par d'importantes modifications socio-anthropologiques générant une grande inquiétude collective sur l'avenir, ce que d’aucuns ont appelé « un nouveau Malaise dans la civilisation » ou Mal-être contemporain (KÄ̈S, 2012).

Dans le cadre de cet article, après quelques rappels épidémiologiques et juridiques permettant de cerner la problématique en cause, j'évoquerai rapidement les grandes caractéristiques cliniques et psychopathologiques des adolescents agresseurs sexuels. Puis l'exposé d'un cas d'adolescent violeur rencontré dans un cadre expertal m’amènera à proposer quelques jalons théoriques relatifs à la place de l'agir dit « sexuel » dans le temps de l'adolescence et ses rapports à la problématique de dépendance ; enfin je terminerai par quelques propos cursifs sur les incidences thérapeutiques posées par la compréhension de ce type d'agirs, inscrits dans ce que nous avons à Paris et à Lyon appelé « les cliniques de l'extrême » (ESTELLON, MARTY, 2012). Celles-ci tout à la fois réinterrogent les modèles de compréhension traditionnels (la perversion, la psychopathie) et questionnent les relations complexes entre l'intrapsychique et l'intersubjectivité, les différents niveaux de l'intersubjectivité, d'où des révisions importantes quand aux modèles thérapeutiques.

\section{SITUATION JURIDIQUE ET ÉPIDÉMIOLOGIQUE DE LA QUESTION}

Les mineurs représentent aujourd'hui 1/4 des auteurs des violences sexuelles judiciarisées qui comprennent les viols, les agressions sexuelles stricto sensu, l'exhibition sexuelle et le harcèlement sexuel. ${ }^{1}$ Notons qu'il s'agit de catégories judiciaires et non psychopathologiques. Ce chiffre est à comparer à d'autres plus généraux : les mineurs représentent environ $18 \%$ des auteurs de délits et de crimes en France, chiffre assez stable depuis les années 2000 : la délinquance

\footnotetext{
${ }^{1}$ Viol : toute acte de pénétration sexuelle, de quelque nature qu'il soit, commis sur la personne d'autrui par violence, menace, contrainte ou surprise ; agression sexuelle : toute atteinte sexuelle commise avec violence, contrainte, menace ou surprise.
} 
des mineurs est certes en augmentation assez régulière sur le plan quantitatif (le nombre de personnes incriminées) mais elle n’augmente pas plus que celle des majeurs, du moins faut-il différencier les types de délinquances. En effet elle augmente plus vite dans le registre des atteintes aux personnes (les agressions) et plus spécifiquement celui des atteintes sexuelles où les mineurs, essentiellement des adolescents (13-18 ans), sont donc surreprésentés. Ce premier fait «brut » interroge ainsi le lien entre l'adolescence (même si celle-ci ne « s'arrête » pas à 18 ans), la propension à l'agir violent, et ce qui a priori peut être considéré comme une forme de lien entre agression et sexualité.

Deuxième constat clinique, à ma connaissance non évalué sur le plan épidémiologique, mais régulièrement évoqué dans la littérature spécialisée (CIAVALDINI et coll., 2013) : la plupart des auteurs d’agressions sexuelles (AAS), quelles qu'elles soient, disent, quand ils veulent bien le reconnaître, avoir débuté leurs agressions à l'adolescence ou à la post-adolescence : à nouveau est questionnée la place de l'adolescence en tant qu'âge, mais également en tant que processus achevé ou inachevé et de l'émergence des problématiques de dépendance qui la caractérisent, dans la genèse des agressions sexuelles.

Enfin dernier constat, selon les études entre 50 et 75\% des AAS disent avoir été victimes d’agressions sexuelles pendant leur enfance, préadolescence ou adolescence, événement à replacer dans le contexte d'un environnement primaire défaillant dans ses fonctions protectrices : l'agression n'est que la face émergée de l'iceberg, le traumatisme sexuel (chaud) cache le traumatisme narcissique (froid), celui des carences et défaillances de l'environnement. Il sera alors intéressant de comprendre les enchainements qui amènent un sujet à ne pouvoir élaborer psychiquement et à répéter activement à l’adolescence ce qu'il a souvent subi passivement.

\section{QUI SONT LES AGRESSEURS SEXUELS À L'ADOLESCENCE ?}

Pour répondre à ces questions il faut maintenant présenter les caractéristiques psychocriminologiques des adolescents auteurs d’agressions sexuelles. Je mêlerai mes propres travaux de recherche, pour certains déjà présentés au Brésil (CHAGNON, 2008, 2009), constitués à partir d'une clinique expertale, aux constats effectués par d'autres auteurs (ROMAN, 2012 ; CIAVALDINI et coll., 2013). Un mot sur la psychocriminologie psychanalytique : celle-ci vise l'étude du fait délinquant ou criminel et pour ce faire elle englobe et dépasse la seule dimension psychopathologique : elle s’intéresse à la clinique du sujet (histoire, état psychique, caractéristiques de personnalité), aux rapports entre l'auteur et sa victime (âge, sexe, liens éventuels, etc.), au contexte de survenue, mais également à la dimension comportementale, « opératoire » du fait criminel. C'est en effet 
dans les modalités les plus concrètes du crime que se « décharge » l'excitation non mentalisée, mais également se « dit » par l'agir « quelque chose » de la problématique traumatique souvent non subjectivée, non symbolisée, mais en attente d'une contenance et d'une réflexion par l'environnement, elle est donc potentiellement messagère même dans ses aspects les plus destructeurs et désobjectalisants (ROUSSILLON, 1999, 2010).

Du point de vue de la justice la question de l'évaluation psychocriminologique des adolescents auteurs d'agressions sexuelles (AAAS) est d'importance car on sait que certaines conduites sexuelles « déviantes » peuvent n'être qu'un accident de parcours, voire une manifestation un peu bruyante de sexualité adolescente encore mal dégagée de ses aspects de sexualité infantile, perverse polymorphe par définition, ou au contraire le point de départ d'une conduite déviante répétitive à l'âge adulte, dont la composante narcissique-identitaire est maintenant bien connue, en particulier depuis les travaux de C. Balier (1996), A. Ciavaldini (1999), ou encore R. Coutanceau (2010).

Il est habituel de distinguer, en ce qui concerne les agressions sexuelles à l'adolescence, les viols individuels - à l'égard d'enfants ou de jeunes femmes - des viols collectifs, ces derniers représentant environ $60 \%$ des cas contre $40 \%$ pour les premiers. Selon C. Legendre (2003), les viols collectifs seraient à situer dans une dynamique psychique propre à l'adolescence, et ce dans un contexte de dysfonctionnement parental, de carences identificatoires et de discontinuités biographiques. Ils répondraient à une recherche agie de l'identité sexuelle chez des sujets pour qui, face à l'incertitude adolescente de la sexuation, la fragilité narcissique nécessiterait un passage à l'acte devant le groupe pour conforter une identification sexuelle masculine. Le groupe aurait un effet désinhibant et fournirait, sur un mode violent et transgressif, une possibilité d’affirmation de soi et d'identification par la domination-possession de la victime, souvent très proche des auteurs. Une dimension initiatique apparentée aux rituels de passage a pu être évoquée. Sans banaliser le phénomène, il s'agirait avant tout d'un phénomène de groupe qui ne concerne pas que les quartiers difficiles, et la plupart des sujets concernés n’auraient pas eu individuellement les mêmes comportements.

Dans ma pratique j'ai eu l'occasion de rencontrer plusieurs adolescents ayant agi en « réunion », dont un cas de figure inhabituel puisqu'il concernait deux frères abusant de leur sœur. Si ces derniers relevaient d'un contexte socio-familial très défaillant où l'incestualité caractérisait les relations familiales, les autres adolescents relevaient pour certains de familles aisées sur le plan socioculturel et économique. Les agressions sexuelles en cause, moins violentes qu'indélicates et prestantes à l'égard des jeunes filles agressées, apparaissaient ponctuelles et sans réel potentiel de répétition même si leurs victimes étaient méprisées et leur altérité faiblement reconnue. S'ils ne niaient pas leurs actes ressortant de circons- 
tances singulières et complexes où la seule dimension psychopathologique était insuffisante à expliquer le passage à l'acte (groupe, foule, festivité, alcoolisation, utilisation de drogues, etc.) ils en niaient en revanche le caractère de contrainte ou de surprise, évacuant facilement leur propre pulsionnalité pour la projeter sur le groupe ou la victime. Pour certains d'entre eux il s'agissait de leur première expérience sexuelle, initiatique donc, pour d'autres les agressions s’inscrivaient dans un activisme sexuel peu scrupuleux prolongeant une instabilité motrice et affective préalable : les filles étaient considérées comme des objets de consommation rapide et jetable, évitant ainsi la peur récurrente d’un enfermement dans une liaison stable, ce qui correspondait à la mentalité «machiste » des groupes qu'ils fréquentaient, mais également de leurs propres familles.

Les agressions sexuelles et viols individuels commis par un adolescent seul relèvent selon les chercheurs, d'une diversité de conduites telle qu'elle en interdit toute généralisation. C'est dans ces cas qu'on rencontrerait les perturbations les plus importantes dans le processus d’adolescence, spécialement le travail de séparation-individuation et de désidentification d'avec les objets primaires, subsumé aujourd'hui sous le vocable de travail de subjectivation (CAHN, 2006). Le processus d’adolescence génère des angoisses dépressives ou de néantisation, angoisses colmatées par les « recours à l'acte » selon une terminologie proposée par C. Balier (1996).

Je retrouve des constats semblables : du point de vue clinique la grande diversité des situations empêche effectivement la généralisation, mes cas balayant des variations de la normale au pathologique le plus grave. Certains renvoient à des défaillances éducatives parentales manifestes alors que ces sujets, en début d'adolescence, ne présentent pas de troubles psychopathologiques majeurs, ce qui ne signifie pas qu'il faille banaliser ces actes, car non reconnus ils peuvent devenir organisateurs de l'adolescence à venir. Les autres (dont 4 cas d'incestes frère-sœur) sont des sujets déjà perturbés psychiquement à des degrés divers : troubles de la personnalité limite ou borderline, psychopathies avec ou sans aménagements pervers, prépsychoses, un cas de psychose infantile.

Si dans leur histoire on retrouve souvent la notion d'un viol ou d'une agression sexuelle subie, ou encore une imprégnation séductrice chronique, ces événements ne prennent sens que rapportés au contexte familial ou social dans lequel ils ont eu lieu : ce sont en effet les liens tendres qui ont échoué à structurer le sujet selon une topique interne constituée, clairement différenciée de son support externe, vectorisée par l'EEdipe, apte à supporter psychiquement l'angoisse et les conflits inhérents au développement pulsionnel. Les traumatismes précoces ont grevé la voie mentale (préconsciente), défensive et élaborative, dès lors peu apte à retenir et traiter l'excitation pulsionnelle. Le couple représentation/ perception est déséquilibré, ces sujets restent dépendants des régulations externes, 
ils surinvestissent la perception, les recours sensori-moteurs et l'agir comme voie d'écoulement des tensions et restent donc dépendants de l'environnement objectal ou non, convoqué pour éprouver, contenir et au mieux transformer le message contenu dans l'agir.

A partir du constat selon lequel les $3 / 4$ des AAAS (en réunion ou seuls) faisaient état d'une instabilité ou hyperactivité infantile j’ai déjà émis l'hypothèse selon laquelle cette hyperactivité, que j’ai proposé de comprendre comme une « hypo-passivité »(CHAGNON, 2009), mutait progressivement en caractère narcissique-phallique dont les potentialités agressantes, pénétrantes ne s'exprimaient qu'au moment de l'adolescence dans un renversement passif/actif bien connu et typique de la lutte contre la dépendance. L'idée principale qui en découle est que l'acte d'agression dite sexuelle est en fait peu sexuel : il vise moins la satisfaction pulsionnelle sexuelle (la plupart des viols n'aboutissent pas à une éjaculation) que la recherche du calme psychique (d'où un potentiel de « récidive », c'est-à-dire de réitération), et la restauration narcissique, voire le colmatage en urgence d'une angoisse identitaire (néantisation, dépersonnalisation) générée par la situation, le contexte relationnel sollicitant massivement la problématique de dépendance irrésolue. La sexualité n’est pas l'objectif, le pénis est le moyen, l’arme vengeresse d'un processus narcissique-identitaire. Cette perspective permet d'articuler deux dimensions débattues, celles des rapports, non contradictoires de mon point de vue, entre la dynamique et l'économique, le sens et la force. Elle permet également de réinterroger le modèle classique de la perversion entaché d’idéologie et de dérive contre transférentielle négative face à ces sujets. Par des voies un peu différentes F. Neau (2005) a aboutit à des conclusions proches en évoquant un «masculin maniaque » en décalque du « féminin mélancolique » décrit et théorisé par C. Chabert (2003).

\section{CLINIQUE DE LA VIOLENCE SEXUELLE}

Un cas « extrême », comme en révèle souvent la clinique expertale qui confronte, davantage qu'en psychiatrie, à l'inhumain, illustrera mon propos. Je rencontre Christophe, jeune adulte de 20 ans, en expertise psychologique dans le cadre de sa mise en examen pour viol : il a en effet violé une amie d'enfance qu'il n'avait pas revue depuis plusieurs années. Niant dans un premier temps, il finira par reconnaître ce viol et s'accusera même de l'avoir prémédité, ce qui paraît peu probable.

Christophe est le premier enfant d'une jeune mère (17 ans) dite « cas social », né d’un père inconnu, sa mère n’ayant jamais voulu lui dire qui il était. Il porte donc dans un premier temps de sa vie le nom de sa mère et se construit une version selon laquelle ce père, dès lors grandiose, pourrait être un meurtrier incarcéré 
au moment où sa mère s'est retrouvée enceinte. Comme cette jeune mère était incapable de l'élever, négligente et de surcroît maltraitante, Christophe lui sera retiré vers l'âge de 3 ans et placé dans une famille d'accueil en même temps qu'une demi-sœur issue d'un autre homme. Celui-ci deviendra le beau-père de Christophe et, bien qualcoolique et maltraitant lui-même, il sera pourtant idéalisé pour lui avoir donné son nom. Christophe ne se remettra cependant jamais de cet arrachement d'une mère qu'il déteste mais ne peut pas quitter : J'arrive pas à la comprendre et je suis souvent en conflit avec elle. Elle parle trop, elle prend ses enfants comme des puzzles, les rassemble et les fait se disputer pour savoir qui l'aimera le plus [...] J'avais l'impression d'être enlevé de ma mère, je recherchais l'amour, il n'y avait que moi, il fallait qu'on rebouche ce trou là, on m’avait enlevé à ma mère.

Enfant déjà agité, l'adolescence verra une éclosion de troubles du comportement (agitation, agressivité dans le cadre scolaire et de la famille d’accueil) du fait de sa faible résistance aux excitations externes : Je suis très influent, je partais au quart de tour, je n'étais pas violent physiquement mais coléreux. Je pars très vite. Je suis pas violent mais il faut me laisser tout seul dans un coin. Quinze personne ça dégénère, je m’amuse, je déconne. Je suis un garçon très influent, on me demande de faire quelque chose je le fais. Maintenant j'ai changé, je me suis endurci. On retrouve également derrière ces troubles du comportement un refus conscient de la dépendance pourtant massive sur le plan inconscient, ainsi qu'une quête paternelle majeure habituelle dans ces cas de figures : J'aurais voulu être occupé toute la journée. Il fallait qu'on s’intéresse à moi, sinon je faisais la tête, je refusais, je redevenais un gamin de 5 ans [...] Je me sentais pris entre ma mère et ma nourrice, j'étais déséquilibré. On remonte et on retombe bien vite [...] À 19 ans, je montais sur les genoux de mon beau-père, je lui faisais des bisous. Ce beau père lui portera pourtant un coup de couteau et Christophe m'exhibera en relevant son pull une longue cicatrice...

Christophe sera suivi en pédopsychiatrie à l'adolescence pour ces troubles du comportement et médicamenté (neuroleptiques), l'éventualité de troubles psychotiques étant envisagée. «Serré » par un juge des enfants, il est orienté dans une ville voisine en internat et peut malgré tout investir une scolarité débouchant, après un passage par l'éducation spécialisée (SEGPA), sur différents diplômes professionnels (CAP, BEP puis BAC professionnel) et enfin un contrat d'embauche. Sur un coup de tête dont la dimension masochiste d'auto-sabotage face à la menace identitaire de dépendance est plus qu'évidente sauf pour l'intéressé, il laisse tout tomber, au moment où son employeur lui propose un CDI, et ce pour se rapprocher de sa mère, sirène mystifiante lui laissant miroiter ainsi que son beau-père des retrouvailles idéalisées. À 19 ans, en se rapprochant géographiquement d'eux qui l'ont appelé pour disqualifier ses engagements et lui promettre un travail jamais entrevu, Christophe rompt avec son foyer éducatif, avec sa petite amie du même âge, avec son employeur prêt à l'engager, écourte son contrat de jeune majeur avec l’ASE, cesse sa prise de traitement médicamen- 
teux. Il finit par se retrouver au chômage, se met à boire et se met en ménage avec une femme de quinze ans son aînée, elle-même mère de quatre enfants.

C'est au décours d'une brouille avec cette femme où elle rompt avec lui, tout en le gardant sous son toit, que le viol aura lieu. La jeune femme violée du même âge que lui était une amie d'enfance qu'il n'avait pas revue depuis plusieurs années et qui avait la double caractéristique de lui ressembler sur le plan psychique (phobie de la séparation) et de le protéger alors qu'ils étaient ensemble à l'école primaire puis au collège. Il tente de reprendre contact avec elle dans la semaine qui suit la rupture d’avec sa concubine dont la composante maternelle est plus qu'évidente. Lors des retrouvailles avec son amie d'enfance, il se laisse aller à évoquer sa souffrance liée à cette rupture, elle a alors un geste tendre de rapprochement et de consolation : elle le prend par l'épaule. C'est alors que tout bascule : Pour moi, je sais pas qu'est ce qui a fait que ça c'est produit. J'arrive pas à me comprendre. C'est comme s'il y avait eu un démon dans mon corps. Je suis pourtant pas violent. Après il y a eu un déclic dans ma tête et je suis redevenu normal.

Qui est Christophe quand il agresse (viole) et qui est sa victime à ce moment là ? L'allusion au démon (confirmée par la victime qui s'est retrouvée brutalement face à quelqu'un d'autre, avec un regard autre) n'est pas une tentative de se disculper, elle recouvre bien une réalité psychique complexe, maintenant bien cernée, celle d'un dédoublement de la personnalité, une abolition subjective, le sujet se dépersonnalisant pour laisser la place à des identifications archaïques, en l'occurrence aux imagos parentales omnipotentes, en particulier à l'agresseur, pour éviter le brusque retour des traumatismes précoces. Dans ce viol, comme dans beaucoup de cas semblables, la douleur de la perte de sa concubine réactive en après coup la perte maternelle précoce inélaborable, le laissant dans une passivité et une dépendance insupportables, que met à jour le geste consolateur de son amie. Le recours à l'acte vient interrompre ce que C. Balier (1996) appelle un «péril d'inexistence » et mettre fin à un bref moment de catastrophe psychotique devant l'explosion, la rupture du déni-clivage des traumas narcissiques précoces réactivés par la confrontation à son amie consolatrice. Plusieurs moments se condensent alors :

- une rupture identificatoire empathique à son amie qui figure tant un double narcissique féminin (sa sœur dira t-il) qu'un objet d'étayage protecteur,

- puis il semble se dépersonnaliser, entrer, via ce que j’appelle une identification projective à l'agresseur (CHAGNON, 2011), dans la peau de ses imagos omnipotentes : père imaginaire grandiose, beau père idéalisé, et, nous le comprendrons plus tard, le violeur de son enfance,

- parallèlement son amie perd son statut de sujet autonome, son altérité, elle devient un objet ustensilitaire dans laquelle il projette, toujours sur un mode identificatoire, différentes parties de lui-même, en particulier sa partie 
féminine-passive, souffrante, maltraitée, mais également l'image maternelle de laquelle il tire vengeance par la terreur qu'il lui impose, renversant ainsi le sens des excitations traumatiques subies dans la petite enfance.

Face à un éprouvé d'envahissement traumatique, de menace d'anéantissement psychique, en se dépersonnalisant et en désobjectalisant sa victime, il opérerait un mouvement de récupération narcissique-phallique par l'usage du sexe comme moyen d'agression. Comme souvent, il s'agit moins de sexualité et de plaisir, que de narcissisme et de vengeance : la violence destructrice prend le pas sur le plaisir érotique (pénétration violente sans éjaculation). Par la pénétration active et violente l'agresseur tenterait de faire entrer, pénétrer de force, et vivre à l’autre ses propres éprouvés de passivation mortifère.

A un niveau légèrement plus évolué, il s'agit également de (mal)traiter les angoisses de perte. Christophe apparaît toujours comme un enfant englué dans sa mère, à moins qu'elle ne soit engluée en lui (BALIER, 1996), dont il cherche à se défaire impérativement pour exister tout en la gardant (il ne semble ni pouvoir la rejeter ni pouvoir l'intérioriser, en faire un objet interne fiable), un enfant qui se sent exclu, arraché à cet objet maternel sans pouvoir s'en séparer faute de cette assise paternelle quêtée et rejetée tout à la fois : «Après le jugement, je décide de quitter X (sa ville), non, quitter ce quartier là, mettre un mur et qu’on me laisse tranquille. Je fais une croix sur ma famille, ma mère me met trop dedans les ennuis. »

Passé un temps de déni, il reconnaîtra son geste et s’accusera même ultérieurement de l'avoir prémédité, ce qui ne correspond ni à la dynamique ni au contexte de l'acte mais constitue une manifestation d'accusation mégalomaniaque, laissant ouverte la question d'une possible évolution mélancolique, faute de capacité à élaborer la perte. Laissé en liberté conditionnelle, Christophe reprendra la vie commune avec sa compagne vivant de nouveau l'amour fou : On va se marier bientôt et on essaie de mettre un enfant en route. Elle veut m'offrir le mien, mon enfant, le mien. Il doit alors percevoir ma désapprobation que je ne cache pas et il fait marche arrière : Je me pose quand même des questions, comment intégrer un enfant au milieu de quatre enfants. ̀̀ 20 ans, on est pas mûr à cent pour cent, mais ma femme ne comprend pas, pour elle c'est une preuve d'amour, ça prouve si je lui fais un enfant que je veux passer ma vie avec. Un enfant c'est pas un jouet qu'on casse...

J’attirerai l'attention des autorités judiciaires sur les potentialités de récidive. La liberté conditionnelle sera assortie d'une obligation de soins que Christophe ne suivra pas. Il sera incarcéré un an plus tard (après l'expertise), après avoir tenté de violer une jeune voisine. Lors de son procès, il reconnaîtra avoir agressé sexuellement depuis son adolescence de nombreux enfants dont ceux de sa concubine et l'un de ses jeunes demi-frères, âgé de 4 ans au moment des faits. Il dira avoir été de nombreuses fois envahi par, et avoir dû lutter contre, l'idée de tuer quelqu'un. Nous apprendrons qu'à l'âge de 4 ans, lors des retours chez sa mère, il était violé par l'un de ses oncles... Ces actes s'étaient réellement 
produits, l'oncle en question ayant été jugé dix ans auparavant par la même cour d’assises. Il n’avait parlé d’aucun de ces événements lors de l'expertise et l'instruction. Enfin, sa mère dévoilera et lui apprendra à la barre qu'il aurait été issu d'un viol incestueux proféré par cet oncle, son frère aîné à elle... La boucle était bouclée. Christophe a été condamné à douze ans de réclusion et à cinq ans de suivi socio-judiciaire comportant une injonction de soins : il se mit alors en colère devant le jury, contestant non la peine de prison prononcée mais le suivi socio-judiciaire ultérieur.

Sur le plan étiopathogénique, on retrouve des aspects malheureusement classiques : la problématique transgénérationnelle incestueuse, l'environnement primaire impitoyable, les traumatismes narcissiques (noyau froid) et sexuels (noyau chaud) indissociables, les identifications narcissiques. La violence des interactions primitives n'a pas permis que s'élaborent des identifications introjectives (assimilées, digérées) post-œdipiennes stables à des objets clairement différenciés et l'adolescence a vu la prise en masse des identifications (incorporations) aliénantes aux imagos omnipotentes (gobées tout cru), dont l'identification projective à l'agresseur, obturant ainsi le travail de mutations décisives, d’appropriation subjective novatrice que l'adolescence comporte dans les bons cas.

\section{DÉPENDANCE ET PASSAGE À L'ACTE : PROPOSITIONS THÉORIQUES}

Proposons maintenant quelques propositions théoriques sur les relations entre la problématique de dépendance et ce type d’agir «sexuel » qui peut être considéré comme un aménagement défensif de celle ci. Ainsi, comme nombre de collègues du CILA, mes propositions s'inscrivent dans une tentative de repenser la théorie des agirs. Les agirs ont du sens au double sens de signification (non symbolique) et de direction, d’adresse à l’autre, fut-il placé sous emprise ou désobjectalisé, privé de son altérité, atteint dans sa chair voire parfois malheureusement dans sa vie. Cela s'inscrit dans une tentative contemporaine de décrire et théoriser un langage du corps et de l'acte à l'adolescence, dont les conséquences thérapeutiques sont majeures puisqu'elles en appellent à l'utilisation de la dépendance à la réalité externe et à l'environnement élargi (JEAMMET, CORCOS, 2003) pour traiter ce qui de la réalité interne cherche à se dire par le corps, l'acte, et donc le passage à l'acte sexuel.

Il n'est pas question ici de considérer toutes les agressions sexuelles sous le statut des toxicomanies et addictions même si nous savons qu'il est possible de parler de «toxicomanie sans drogue » (Fénichel) et d'étendre ainsi le spectre des conduites addictives. C'est davantage sous l'angle de l'émergence des problématiques de dépendance et de leurs aménagements à l'adolescence que je me 
situe, et ce en référence aux travaux fondamentaux de $\mathrm{Ph}$. Jeammet, seul ou en compagnie de M. Corcos (2003).

Selon les auteurs, que je cite et résume largement, l’adolescence « est une période charnière qui impose un changement de l'équilibre entre dedans et dehors comme de la relation avec les parents et avec le corps propre, (elle) remet en cause le rapport narcissisme — relation objectale, dépendance — autonomie. Il y a de ce fait, une fragilisation du monde psychique interne lequel est fortement sollicité aussi bien dans ses assises narcissiques que dans ses investissements objectaux. Le rapport à la réalité externe s'en trouve sensiblement modifié. Ce changement contribue à conférer une portée heuristique, dans la saisie des comportements à cet âge, à la notion de dépendance comme contre investissement de la réalité interne par la réalité externe » (2005, p.1).

Toujours selon les auteurs, toute la psychopathologie de l'adolescence peut ainsi être vue comme un aménagement de la problématique de dépendance. Dans les cas les plus graves, pour les raisons précitées, du fait de la massivité des traumatismes précoces ayant grevé la construction de « la voie mentale longue », celle de la symbolisation, de la liaison représentation/affects sous l'égide du principe de plaisir modifié en principe de réalité, celle qui donne lieu au plaisir de penser, de désirer, prélude à l’action spécifique, les régulations s'effectuent essentiellement par les recours au comportement et à la sensori-motricité, privant en retour les possibilités d'intériorisation, d'identification introjective, de différenciation et de subjectivation qui viennent habituellement nourrir le Moi et constituer l'identité. L'adolescence par ses «tâches développementales » (intégrer une nouvelle identité sexuée, travail de séparation-individuation, de subjectivation) vient mettre à l'épreuve les assises narcissiques, les différenciations dedans/dehors, et au-dedans entre instances (Moi, Surmoi-Idéal du Moi), en bref l'inélaboré, l'inachevé de l'enfance. La balance entre investissements narcissiques et investissements objectaux est alors déséquilibrée au risque d'un antagonisme voire d'une rupture dite narcissico-objectale.

La crainte majeure de tout adolescent est celle de la perte de contrôle de l'excitation et de la confrontation aux désirs passifs sollicités tant par les pulsions que l'objet. La question de la maîtrise du lien et du contrôle de la distance objectale devient centrale. Quand l'équilibre narcissique est trop massivement dépendant des appuis externes, au détriment des investissements internes, alors des phénomènes de réaction en miroir, de renversement passif/ actif, subi/ agi sont sollicités pour protéger l'identité : par exemple le sujet cherche à rendre dépendant de lui l'objet dont il dépend, il cherche à lui faire vivre ce que lui-même a vécu ou vit passivement pour en retrouver la maîtrise. C'est à ce niveau que jouent la relation et les mécanismes d'emprise, comme aménagement de la dépendance, emprise à l'égard de l'objet de dépendance, mais également à 
l'égard de la réalité externe, celle du percept et des sensations, au détriment de la réalité interne, celle des représentations et affects : le plaisir d’emprise prime sur le plaisir de la satisfaction.

On peut reprendre le cas Christophe sous cet angle, auquel j'y ajoute l'importance de la dimension identificatoire primaire comprise dans le passage à l'acte sexuel (CHAGNON, $2011 ; 2013)$. La reviviscence des angoisses dépressives de séparation amplifiées par le $2^{\text {ème }}$ temps de l'Edipe, surtout quand il n'a pas été structurant (Edipe sans complexe), fait resurgir les premières angoisses ou excitations traumatiques mal surmontées car insuffisamment symbolisées et subjectivées. Les mécanismes archaïques sont alors remobilisés par voie rétroactive avec le risque de devenir déterminants. Du fait des vicissitudes historiques (trop peu de satisfactions tendres, emprise parentale dédifférenciatrice, intromission d’objets transgénérationnels, deuils non faits, gelés, enkystés, etc.) «le psychisme devient prisonnier d'incorporats hostiles ou hyperexcitants installés au cœur de l'être » (CAHN, 2002, p.120-121). Quand le déni, le clivage et l'idéalisation sont débordés et que les affects primitifs risquent de resurgir alors les mécanismes d'identification projective accompagnent les passages à l'acte qui permettent tant d'évacuer du psychisme la tension interne que de faire vivre à l'autre l'innommable, ce qui peut épargner une décompensation psychotique. C’est en effet cette dimension d'identification projective, intrusive, au sens donné par Bion, qui me parait caractériser, au-delà de l'emprise, le passage à l'acte sexuel, l'agir pathologique.

\section{CONCLUSIONS : INCIDENCES SUR LE SOIN}

Cette modélisation du passage à l'acte a des incidences assez précises quand à l'organisation des soins et des traitements individuels, groupaux ou institutionnels, à médiation. Ici le travail thérapeutique ne peut plus se centrer sur la seule réalité interne, à partir de laquelle on interpréterait l'utilisation de la réalité externe. Tant les spécialistes de l'adolescence que ceux de la criminologie se rejoignent à ce niveau ; il s'agit d’aménager la réalité externe de façon à renforcer la capacité d'élaboration psychique, de jeu et de déplacement, et secondairement de travailler sur la reconnaissance de la réalité interne. C'est l'ensemble du dispositif de soin qui doit être repensé en référence à cette compréhension du (dys)fonctionnement mental, à la dépendance perceptivo-motrice de ces sujets, de la consultation initiale qui ne peut plus se contenter d'attendre une illusoire demande (CHAGNON, HOUSSIER, 2012) aux modalités thérapeutiques nécessairement organisées par la nécessité de survivre à plusieurs à la destructivité. Avec ces sujets clivés, l'essentiel du travail des soignants (la thérapie individuelle n'est pas conseillée au moins d'emblée) sera de mettre en place et veiller au 
maintien d'un cadre collectif qui contienne la multiplicité des facettes d'une même personne et d'accepter, dans la diversité des liens et des échanges avec les collègues, d’endosser les rôles parfois désagréables que l’adolescent dépose provisoirement en eux par identification projective, de façon à lui permettre en les lui « réfléchissant », de les réintrojecter et se les réapproprier autrement. Le contre transfert, y compris dans ses dimensions somatiques et agies, est alors l'outil privilégié pour entendre et transformer l'innommable.

Recebido em 17/1/2014. Aprovado em 4/2/2014.

\section{RÉFÉRENCES}

BALIER, C. (1996) Psychanalyse des comportements sexuels violents. Paris : PUF. CAHN, R. (2002) Les identifications à l'adolescence, in Monographies de la revue française de psychanalyse, 2002 (Identifications). Paris : PUF, p.111-125. . (2006) «Origines et destins de la subjectivation », in RICHARD, F., WAINRIB, S. (Orgs.) La Subjectivation. Paris : Dunod, p.7-18.

CHABERT, C. (2003) Féminin mélancolique. Paris : PUF.

CHAGNON, J.-Y. (2008) Les agressions sexuelles : un aménagement des troubles narcissiques identitaires. Paidéia. Cadernos de Psicologia e educação, v.18, n.41, Ribeirão Preto, p.495-515.

(2009) A agressão sexual na adolescência: um destino da hiperatividade? (L'agression sexuelle à l'adolescence : un destin de l'hyperactivité?). Agora — Estudos em Teoria Psicanalítica, IP — UFRJ, v.XII, n.2, jul.-dez. 2009. Rio de Janeiro, p.275-290.

. (2011) Identification à l'agresseur et identification projective à l'adolescence. A propos d'un cas. Topique, n.115, p.127-140. HOUSSIER, F. (2012) L'illusoire attente de la demande. Adolescence (Enfermement II), n.4, p.919-933.

(2013) Qui est qui, qui agresse qui ? Traumatisme, passage à l'acte et identification projective à l'adolescence. Perspectives Psy, n.2, p.134-139.

CIAVALDINI, A. (1999) Psychopathologie des agresseurs sexuels. Paris : Masson. (2013) Violences sexuelles chez les mineurs. Paris : In Press._

COUTANCEAU R., SMITH, J. (2010) La violence sexuelle. Approche psychocriminologique. Paris : Dunod.

ESTELLON V., MARTY, F. (dir.) (2012) Cliniques de l'extrême. Paris : Armand Colin.

FREUD, S. Fuvres complètes. Psychanalyse. Paris : PUF.

JEAMMET, P., CORCOS, M. (2005) Evolution des problématiques à l'adolescence. L'émergence de la dépendance et ses aménagements. 2 ed. Rueil-Malmaison : Doin. KAËS, R. (2012) Le Malêtre. Paris : Dunod.

LEGENDRE, C. (2003) Les adolescents auteurs de viols. Psychiatrie Française, n.2-3, p.104-115. 
NEAU, F. (2005) Masculin maniaque ? Psychologie Clinique et Projective, v.11, p.35-78.

ROMAN, P. (2012) Les violences sexuelles à l'adolescence. Issy Les Moulineaux : Masson.

ROUSSILLON, R. (1999) Agonie, clivage et symbolisation. Paris : PUF.

(2010). Pour introduire la question du langage du corps et de l'acte, in GOLSE, B., ROUSSILLON, R., La naissance de l'objet. Paris : PUF, p.177-189.

Jean-Yves Chagnon

Jean-yves.chagnon@libertysurf.fr 